\title{
Susac Syndrome with Frontal Intermittent Rhythmic Delta Activity (FIRDA)
}

\author{
Danielle Woolridge, Mark Stefanelli, Barbara Hoppe
}

\begin{abstract}
Background: Susac syndrome is a rare condition involving the brain, retina, and cochlea. Electroencephalogram (EEG) findings from published case reports show mainly generalized slowing. Case report: A 30-year-old man presented with acute onset of superior vision loss, unsteady gait, and hearing loss. This was accompanied by short-term memory loss and behavioral and mood changes. MRI showed multiple white matter hyperintensities. The EEG showed frontal intermittent rhythmic delta activity. A diagnosis of Susac syndrome was made and treatment with methylprednisolone, ASA, and Nimodipine was instituted. At one-year follow-up, bilateral hearing loss, mild visual impairment, and mood changes were still apparent. Conclusions: Frontal intermittent rhythmic delta activity can be seen in Susac syndrome.
\end{abstract}

RÉSUMÉ: Un cas de syndrome de Susac avec activité delta rythmique intermittente au niveau frontal. Contexte : Le syndrome de Susac est une maladie rare du cerveau, de la rétine et du limaçon. L'électroencéphalogramme (ÉEG) démontre surtout un ralentissement généralisé chez les cas qui ont été publiés. Observation : Un homme de 30 ans a consulté pour une perte de vision aiguë dans le champ de vision supérieur, un déséquilibre à la marche et une perte auditive. De plus, il accusait une diminution de la mémoire à court terme et des changements de comportement et d'humeur. L'IRM a montré de multiples zones d'hyperintensité dans la substance blanche. L'ÉEG a montré une activité delta rythmique intermittente au niveau frontal. Le diagnostic de syndrome de Susac a été posé et le patient a été traité par la méthylprednisolone, l'ASA et la nimodipine. Au suivi, une perte auditive bilatérale, une légère perte de vision et des changements d'humeur étaient encore présents un an plus tard. Conclusions : Une activité rythmique intermittente frontale peut être observée dans le syndrome de Susac.

Susac syndrome is a rare condition consisting of a triad of branch retinal artery occlusion, hearing loss, and encephalopathy. Less than 100 cases have been reported in the literature since the first cases were described in 1979 by Susac. ${ }^{1}$ Many authors have reported EEG findings in their patients. In this case report of a patient with Susac syndrome, frontal intermittent rhythmic delta activity (FIRDA) was seen on EEG.

\section{CASE Report}

A 30-year-old man presented with sudden superior vision loss in the left eye and unsteady gait. He reported episodes of dysarthria lasting minutes throughout the day. The next day he had decreased hearing in the right ear that progressed to complete deafness bilaterally within a few days. He also described headache, tinnitus, mild short-term memory loss, and aggressive behavior with mood swings. His medical history was previously unremarkable. Neurological examination on admission revealed an ataxic gait. Formal neuropsychological testing did not reveal memory impairment; however, emotional lability was noted during hospitalization. Formal

\footnotetext{
From the Memorial University of Newfoundland, St. John's, NL, Canada. Received January 5, 2006. ACCEPTED IN FinAl FORM June 27, 2006. Reprint requests to: Danielle Woolridge, Memorial University of Newfoundland, 10 Larner St., St. John's, Newfoundland, A1A 5S7, Canada.
} 
ophthalmological examination showed a visual acuity of $20 / 25$ in the right eye and 20/60 in the left eye. Visual field testing showed superior visual field loss in the left eye. Intraocular pressures and slit lamp examination were unremarkable. On dilated funduscopy examination, the right eye was normal; the left eye showed attenuation with sheathing of the inferior branch of the retinal artery as well as resolving cloudy swelling; features suggestive of an inferior branch retinal artery occlusion. Formal audiological examination revealed bilateral sensorineural hearing loss in the low frequency range.

An extensive systemic screen for collagen vascular disease, coagulopathy, infectious, metabolic, and autoimmune causes were negative. Transthoracic echocardiogram and muscle biopsy were also negative. Magnetic resonance imaging (MRI) of the brain revealed multiple white matter hyperintensities on T2weighted images involving the right anterior corpus callosum, left rostrum of the corpus callosum, and periventricular white matter (Figure 1). On T1-weighted images, some lesions enhanced with Gadolinium. Lumbar puncture yielded an elevated total protein of $0.74 \mathrm{~g} / \mathrm{L}$ and a mild lymphocytosis. Oligoclonal banding was negative. The EEG showed FIRDA at a frequency of $2.5 \mathrm{~Hz}$ (Figure 2) and intermittent trains of low to medium amplitude, $2-3 \mathrm{~Hz}$, asynchronous delta waves in the left and right fronto-temporal regions. During sleep, there was activation of focal spikes and sharp waves in the right temporal and parietal regions. A repeat EEG was not obtained.

A diagnosis of Susac syndrome was made based on the branch retinal artery occlusion, sensorineural hearing loss, and encephalopathy. Treatment consisted of methylprednisolone, nimodipine, ASA, intertympanic membrane steroid injections, and grommet insertions.

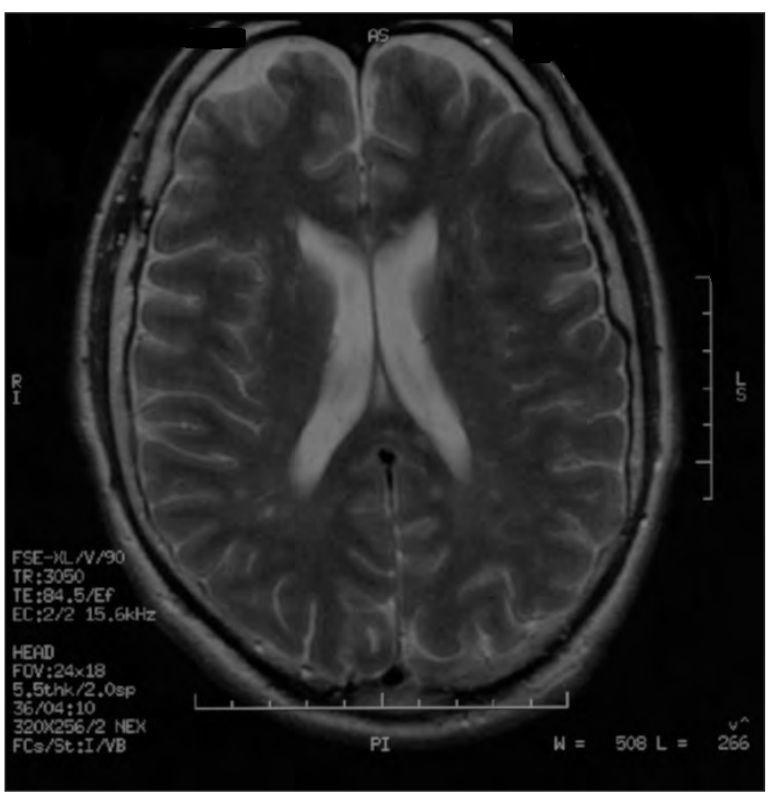

Figure 1: Axial T2-weighted MR image of the brain demonstrating hyperintense lesions in the periventricular white matter and corpus callosum.
At one-year follow-up, visual acuity had returned to 20/20 in each eye. Visual field testing was normal in the right eye but continued to show superotemporal field loss in the left eye. Funduscopic examination yielded residual attenuation of the retinal artery vasculature in the left eye. Significant hearing loss, headache, and mood changes including depression and anger were still problematic. Hearing had improved minimally with treatment but worsened again a few months later. Gait had normalized but the patient had a mild left sided pronator drift (not present initially) on neurological examination. Repeat MRI was unchanged from presentation.

\section{DiscuSSION}

This patient was diagnosed with Susac syndrome (microangiopathy of the retina, brain, and cochlea) based on the clinical triad of branch retinal artery occlusion, sensorineural hearing loss, and encephalopathy. ${ }^{1}$ Other signs and symptoms were present that are commonly found in this disorder including memory loss (46\% of the 46 patients reviewed in 1998), headache $(33 \%)$, psychiatric changes $(41 \%)$, ataxia $(52 \%)$, and dysarthria $(20 \%){ }^{2}$ Hemiparesis $(30 \%)$, hemisensory changes (17\%), incontinence (4\%), and Babinski sign (7\%) have also been described but were absent in the present case. Headache can be a prodromal symptom several months before the onset of other symptoms. ${ }^{1,3}$ The gait impairment that is present in over half of cases may be due to involvement of the eyes, ears, or brain. Although Susac initially believed this to be a disorder of young women, a number of male cases have also been described. ${ }^{1,2}$ There is a female to male ratio of 3 to 1 and an age range of $16-58$ years. ${ }^{4}$ Duration of the illness can range

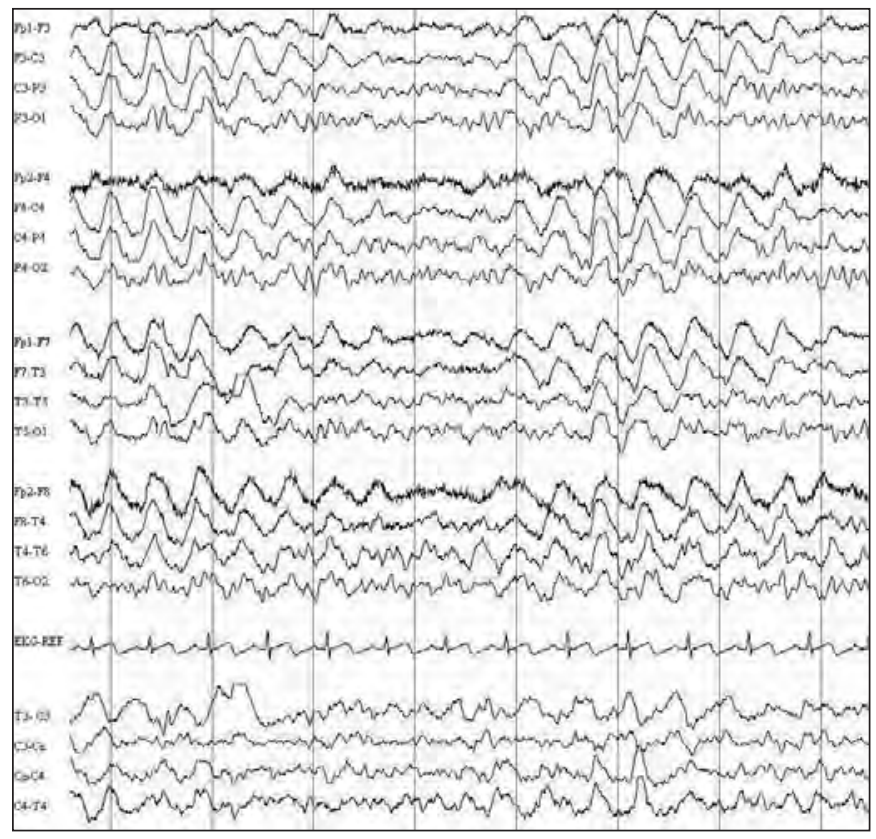

Figure 2: The EEG of a 30-year-old man with Susac syndrome showing FIRDA (longitudinal bipolar montage recorded at $75 \mu \mathrm{Vp}-\mathrm{p}$ ). 
anywhere from 2 months to 11 years with a mean duration of 46.7 months. ${ }^{2}$ The disease is episodic with 1-8 attacks (mean 2.8) before spontaneous remission. Some degree of hearing loss and visual impairment is permanent in the majority of patients. One third of patients are left with a gait disturbance or dementia. ${ }^{3}$

Cerebral spinal fluid examination often contains elevated protein with minimal pleocytosis, usually lymphocytic. ${ }^{1,4}$ The MRI typically exhibits small hyperintense lesions in the white matter, supratentorially, with a propensity for the corpus callosum. Lesions have also been found in the cerebellum, midbrain, and gray matter. Enhancement with Gadolinium can be found in some of the lesions as well as in the leptomeninges. ${ }^{5}$

A hypothesized immunological etiology for the disease is a small vessel vasculitis leading to microinfarcts in the retina, cochlea, and brain. ${ }^{2,6}$ Brain biopsy in nine patients revealed small infarcts from arteriolar occlusions. ${ }^{3}$ It also has been postulated that Susac syndrome may be due to vasospastic dysregulation. ${ }^{7}$ Further research is necessary to further elucidate the etiology of this syndrome.

Because there are so few cases reported in the literature, therapy is based on anecdotal evidence of individual improvement. This is complicated by the fact that the disease has a spontaneous remission after the active phase. A number of different therapies have been tried and recommended. Susac ${ }^{1}$ in 1994 recommended initial treatment with aspirin and nimodipine, and then high-dose intravenous methylprednisolone as second line therapy. His third line therapeutic recommendation was cyclophosphomide. O'Halloran et $\mathrm{al}^{2}$ in 1998 recommended treatment with intravenous methylprednisolone 1 $\mathrm{g} /$ day for 3 days and then oral prednisone at $80 \mathrm{mg}$ a day with a slow taper. Cyclophosphomide was recommended as an adjunct for treatment failure.

Electroencephalogram during the encephalopathic phase of Susac syndrome is most commonly diffusely slow. ${ }^{1,2,4}$ Seizure activity may also be present. ${ }^{3}$ Widespread triphasic waves were reported in one 29 -year-old male. ${ }^{6}$ One patient with prominent, diffuse, slow wave activity without epileptiform activity or lateralization, and another patient with 1- to 2- $\mathrm{Hz}$ bursts from the right frontotemporal region and a slow disorganized background have been reported. ${ }^{8}$ Nonspecific theta dysrhythmia over temporal channels was seen in a 30-year-old male who reportedly had two generalized tonic clonic seizures preceding the onset of other symptoms by 7 years. ${ }^{9}$ This is the only report of clinical seizures associated with the disorder. The authors postulated that the epileptic seizures were the first episode in a multiphasic course of the disease. Two patients reported by Petty et $\mathrm{al}^{10}$ had EEGs showing paroxysmal generalized, bisynchronous, delta activity maximal in the frontal regions (FIRDA).

Our patient also had FIRDA on EEG. Frontal intermittent rhythmic delta activity is a transient rhythmic slow wave discharge with a frequency of 1.5-2.5 cycles per second. The maximum of the activity is found in the frontopolar regions of the brain. ${ }^{11,12}$ The clinical and radiological correlates of FIRDA have been studied throughout the years and it appears to be a non-specific EEG pattern with a large differential diagnosis. ${ }^{13,14}$ It was initially described in association with tumors of the posterior fossa or third ventricle and hydrocephalus. ${ }^{15}$ It was later found in equal frequency in patients with metabolic encephalopathies, deep midline lesions and hemispheric lesions and was thought not to be associated with hydrocephalus. ${ }^{13}$ Metabolic encephalopathy associated with renal insufficiency, hepatic failure, and anoxic brain injury is common in patients with non-structural causes of FIRDA. ${ }^{14}$ Frontal intermittent rhythmic delta activity has been present with delirium and dementia especially in the presence of cerebrovascular disease, head trauma, endocrine diseases such as diabetes mellitus, CNS infection, dementia with Lewy bodies, in patients with a history of brain tumor surgery, and in patients with a history of partial epilepsy. ${ }^{12,16,17}$ It is a transient pattern that transforms to a different EEG pattern within days to weeks. Chronic white matter, basal ganglia, thalamic, and cerebellar ischemic diseases were the most common MRI findings in patients with FIRDA. No correlation was found with midline structural lesion or infratentorial lesions. ${ }^{14,18}$

Watemberg et al. ${ }^{11}$ in 2002 reviewed 22,000 EEG recordings. Frontal intermittent rhythmic delta activity was found in 147 cases and of these, 68 medical records were reviewed. The most common radiological findings were diffuse and localized ischemic brain disease. Patients with FIRDA tended to be elderly with chronic diseases such as hypertension, diabetes mellitus, and renal disease. From these findings, the authors suggested that FIRDA occurs transiently in patients with diffuse brain injury and concomitant metabolic impairment. They also suggested, FIRDA is not epileptic in nature but may be detected post-ictally.

Susac syndrome causes widespread ischemic brain lesions secondary to a postulated small vessel vasculitis. The majority of cases of Susac syndrome reported thus far have had diffuse slowing on EEG. In this case report, and in two patients reported previously, ${ }^{10}$ FIRDA was the predominant EEG abnormality in the absence of a metabolic derangement. Since FIRDA is associated with many causes of diffuse brain disease, more cases of Susac syndrome with FIRDA may be reported in the future.

\section{REFERENCES}

1. Susac JO. Susac's syndrome: the triad of microangiopathy of the brain and retina with hearing loss in young women. Neurology. 1994; 44(4):591-3.

2. O'Halloran HS, Pearson PA, Lee WB, Susac JO, Berger JR. Microangiopathy of the brain, retina, and cochlea (Susac syndrome). Ophthalmology. 1998; 105(6):1038-44.

3. Papo T, Biousse V, Lehoang P, Fardeau C, N'guyen N, Le Tri Huong D, et al. Susac syndrome. Medicine. 1998; 77(1):3-11.

4. Susac JO. Susac's syndrome. AJNR Am J Neuroradiol. 2004; 25(3):351-2.

5. White ML, Zhang Y, Smoker WRK. Evolution of lesions in Susac syndrome at serial MR imaging with diffusion-weighted imaging and apparent diffusion coefficient values. AJNR Am J Neuroradiol. 2004; 25(5):706-13.

6. Saw VPJ, Canty PA, Green CM, Briggs RJ, Cremer PD, Harrisberg B, et al. Susac syndrome: microangiopathy of the retina, cochlea and brain. Clin Experiment Ophthalmol. 2000; 28(5):373-81

7. Flammer J, Kaiser H, Haufschild T. Susac syndrome: a vasospastic disorder? Eur J Ophthalmol. 2001; 11(2):175-9.

8. Monteiro MLR, Swanson RA, Coppeto JR, Cuneo RA, DeArmond SJ, Prusiner SB. A microangiopathic syndrome of encephalopathy, hearing loss, and retinal arteriolar occlusions. Neurology. 1985; 35:1113-21.

9. Szilasiová J, Klímová E. Susac syndrome: retinocochleocerebral vasculopathy. Croat Med J. 2004; 45(3):338-43. 
10. Petty GW, Engel AG, Younge BR, Duffy J, Yanagihara T, Lucchinetti CF, et al. Retinocochleocerebral vasculopathy. Medicine. 1998; 77(1):12-40.

11. Watemberg N, Alehan F, Dabby R, Lerman-Sagie T, Pavot P, Towne A. Clinical and radiologic correlates of frontal intermittent rhythmic delta activity. J Clin Neurophysiol. 2002; 19(6):535-39.

12. Zurek R, Delgado JS, Froescher W, Niedermeyer E. Frontal intermittent rhythmical delta activity and anterior bradyrhythmia. Clin Electroencephalogr. 1985; 16(1):1-10.

13. Hooshmand $\mathrm{H}$. The clinical significance of frontal intermittent rhythmic delta activity (FIRDA). Clin Electroencephalogr. 1983; 14(3):135-7.

14. Fariello RG, Orrison W, Blanco G, Reyes PF. Neuroradiological correlates of frontally predominant intermittent rhythmic delta activity (FIRDA). Electroencephalogr Clin Neurophysiol. 1982; 54(2):194-202.
15. Daly D, Whelan JL, Bickford RG, MacCarty CS. The electroencephalogram in cases of tumors of the posterior fossa and third ventricle. EEG Clin Neurophysiol. 1953; 5:203-16.

16. Kubota F, Ohnishi N. Study on FIRDA and $3 \mathrm{~Hz}$ rhythmic slow wave bursts occurring in the frontal area of epileptic patients. Clin Electroencephalogr. 1997; 28(2):112-6.

17. Calzetti S, Bortone E, Negrotti A, Zinno L, Mancia D. Frontal intermittent rhythmic delta activity (FIRDA) in patients with dementia with Lewy bodies: a diagnostic tool? Neurol Sci. 2002; 23 Suppl 2:S65-6.

18. Watemberg N, Towne AR. Radiologic correlations of frontal intermittent rhythmic delta activity. Epilepsia. 1997; 38 Suppl 4:119. 